\title{
NIHTS: the near-infrared high throughput spectrograph for the Discovery Channel Telescope
}

Edward W. Dunham, Thomas A Bida, Tomas Chylek, Frank Cornelius, Annika Gustafsson, et al.

Edward W. Dunham, Thomas A Bida, Tomas Chylek, Frank Cornelius, Annika Gustafsson, Nicholas Moskovitz, Henry Roe, "NIHTS: the nearinfrared high throughput spectrograph for the Discovery Channel Telescope," Proc. SPIE 10702, Ground-based and Airborne Instrumentation for Astronomy VII, 107023E (6 July 2018); doi: 10.1117/12.2313948 


\title{
NIHTS: The Near-Infrared High Throughput Spectrograph for the Discovery Channel Telescope \\ Edward W. Dunham* ${ }^{* a}$, Thomas A. Bida ${ }^{\mathrm{a}}$, Tomas Chylek ${ }^{\mathrm{b}}$, Frank Cornelius ${ }^{\mathrm{a}}$, Annika Gustafsson ${ }^{\mathrm{c}}$, Nicholas Moskovitz ${ }^{\mathrm{a}}$, and Henry Roe ${ }^{\mathrm{d}}$ \\ ${ }^{\mathrm{a}}$ Lowell Observatory, 1400 West Mars Hill Road, Flagstaff, AZ, USA 86001; ${ }^{\mathrm{b}}$ Thirty Meter \\ Telescope Project, 100 West Walnut Street \#B3, Pasadena, CA USA 91124; ${ }^{\mathrm{c}}$ Northern Arizona \\ University, Flagstaff, AZ, USA 86001; Gemini Observatory, c/o AURA Casilla 603, La Serena, Chile.
}

\begin{abstract}
NIHTS is a first-generation instrument now in use on Lowell Observatory's Discovery Channel Telescope. It is a nearinfrared prism spectrograph of the BASS design featuring high throughput and low dispersion that is intended for observations of faint solar system and astrophysical objects over the YJHK spectral range. An unusual feature is its ability to observe simultaneously with the Large Monolithic Imager, an optical CCD camera, by means of a dichroic fold mirror. This is particularly valuable for time-variable targets such as Kuiper Belt Objects, asteroids, exoplanet transits, and brown dwarfs. We describe its design details and performance both in the lab and on the telescope.
\end{abstract}

Keywords: Discovery Channel Telescope (DCT), Near-IR, spectrograph, Lowell Observatory

\section{INTRODUCTION}

NIHTS is a near-IR prism spectrograph designed to obtain low-resolution spectra with high throughput using Lowell's Discovery Channel Telescope (DCT) ${ }^{1}$. It will primarily be used for observations of faint Kuiper belt objects, near-earth objects, and brown dwarfs, but will likely have many other science applications such as exoplanet transits.

DCT instruments are mounted on the instrument cube that also contains the guiders and wavefront sensors ${ }^{2}$. NIHTS is a small port instrument that was originally intended to be smaller but grew when an Offner relay and cold stop were added and its resolution doubled to take advantage of a HAWAII-1 detector. The instrument has no moving parts but a stepped slit allows the observer to choose among several different slit widths.

The principal design requirements were maximum throughput over a wavelength range from 0.9 to 2.4 microns using a HAWAII-1 1Kx1K HgCdTe detector. The spectral resolution followed from the detector format combined with the slit width selected for the observation. The instrument contains an Offner relay and cold stop, but the cold stop is slightly oversized to ensure that maximum throughput is obtained at the cost of higher thermal background at long wavelengths.

NIHTS also includes a Xenics Xeva-1.7-320 InGaAs slit-viewing camera with associated re-imaging optics. A very unusual feature of NIHTS is that its fold mirror in the DCT instrument cube is a dichroic beamsplitter that allows simultaneous observations to be made at optical wavelengths with $\mathrm{LMI}^{3}$.

The following sections describe the optical design, mechanical design, cryogenics, electronics, software, and realized performance of NIHTS on the DCT. NIHTS has been described in part in a previous SPIE proceedings paper ${ }^{3}$ that was written before NIHTS was completed; we attempt here not to duplicate information in that paper.

Ground-based and Airborne Instrumentation for Astronomy VII, edited by Christopher J. Evans, Luc Simard, Hideki Takami, Proc. of SPIE Vol. 10702, 107023E · @ 2018 SPIE · CCC code: 0277-786X/18/\$18 - doi: 10.1117/12.2313948 


\section{OPTICAL DESIGN}

\subsection{NIHTS optics}

The NIHTS resolution and throughput requirements were best met by a prism spectrograph of the BASS design ${ }^{4}$. This is a double-pass design in which both prism surfaces and the return mirror are powered. These surfaces perform the collimator and camera optics functions without requiring additional surfaces. To fit the higher-resolution version of NIHTS and the Offner relay in a DCT small port envelope we had to add five reflections: two fold mirrors and the three reflections in the Offner relay, but these mirrors are all gold coated and cold so little is lost by adding them.

We selected $\mathrm{ZnS}$ (Cleartran) for the prism material for dispersion reasons. (This is called out incorrectly in Reference 3 as $\mathrm{ZnSe}$.) The maximum thickness available for this material is $44 \mathrm{~mm}$, which limited the amount of angular dispersion that the prism could provide. The required linear dispersion at the detector then defined the path length between the prism and detector. This in turn required two fold mirrors. The entrance and exit windows are the only other transmissive elements. Both are IR-grade fused silica. All of the transmissive surfaces are anti-reflection (AR) coated (see section 2.4).

The slit assembly ${ }^{3}$ is a diamond-machined assembly with a reflective surface at a $45^{\circ}$ angle to the optical axis, made for us by Durham Precision Optics. It features a stepped set of slit widths along the length of the slit so that the slit width can be changed by re-pointing the telescope. Light reflected from the slit face is re-imaged onto the slit-viewing camera (see next section) while light transmitted through the slit propagates into the spectrograph.

As described below the opto-mechanical design was based on precision machining of the optical elements, their mounts, and the optical table to eliminate alignment adjustments except for detector focus and tilt. The design included configurations for room temperature and $80 \mathrm{~K}$, using refractive indices at those temperatures from John Rayner. Zemax does thermal compensation for the "air" spacings based on the thermal expansion coefficient of the material the optics are mounted on, aluminum in the case of NIHTS, but it does not account for the variation in this coefficient with temperature. This was done by hand, using detector focus as the final compensator for remaining uncertainties.

One alignment consideration we overlooked was rotation of the prism about its axis. We didn't specify an index feature on the edge of the prism to define this degree of freedom. To work around this error we determined the location of the thinnest part of the prism by placing it on a rotary table and measuring the height of the glass at $10^{\circ}$ intervals with a dial indicator. A least squares fit determined the position of the thinnest point. We marked it and assembled the prism into its mount in that orientation. A retaining clip was attached to the ground edge of the prism with aluminum tape. This clip was constrained to avoid possible rotation of the prism in its mount over long periods of time.

Focusing and setting the detector tilt were accomplished by moving the detector mounting bracket relative to the optical bench $^{3}$ to minimize the widths of xenon lines across the spectrum so focus iterations required several cold cycles. In the lab the xenon lamp was imaged onto the slit with lenses to create an $f / 6$ beam to mimic the telescope optics. We were able to speed the focus iteration process by observing the line widths as the optical bench cooled down and warmed up. This allowed us to determine the defocus direction and approximate magnitude, and we converged on our current focus after 3 iterations.

The optical layout of the spectrograph is shown in Figure 1. Figure 2 shows spot diagrams for 5 fields separated by 20" along the slit and 8 wavelengths as called out in the legend.

\subsection{Slit-viewing optics}

The slit-viewing optics, shown in Figure 3, are a refractive collimator/camera focal reducer to match the format of the Xenics camera. Figure 4 shows spot diagrams at several field positions. The design uses stock lenses for all but the field flattener, an IR-grade fused silica meniscus lens. The two biconvex lenses are $\mathrm{BaF}_{2}$ to reduce lateral color and the remaining optics are fused silica. Throughput of the optics is also important so they were AR-coated (see Section 2.4).

The slit-viewing optics were focused on the slit in the lab, with the sharpest focus being determined by eye with an uncertainty of about 150 microns. The focus was set at this position with a shim. 


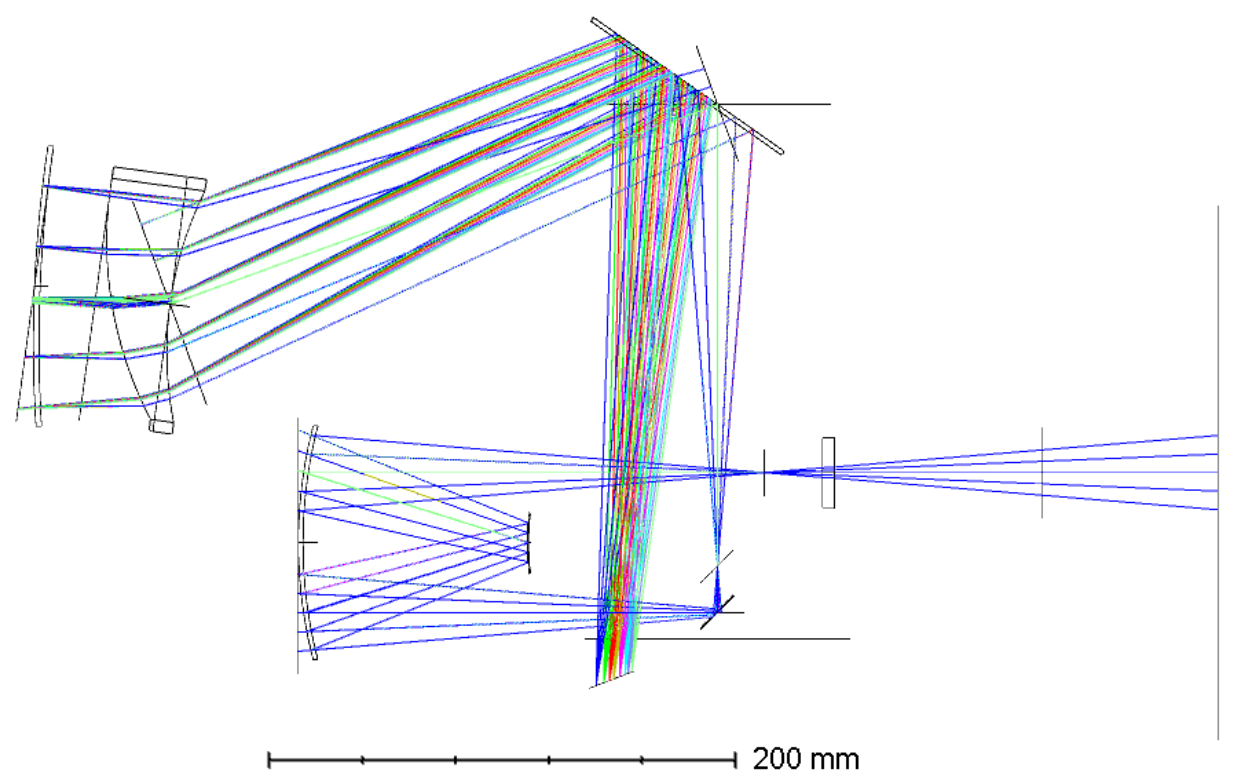

Figure 1. Layout of the NIHTS spectrograph optics. Light from the DCT enters at lower right, proceeds through the Offner relay, off a small fold mirror and focuses at the slit. From the slit it makes a double pass off the large fold mirror and prism, reflecting from the return mirror. The detector is at lower center, tilted significantly from the optical axis. The rays are colored by wavelength.

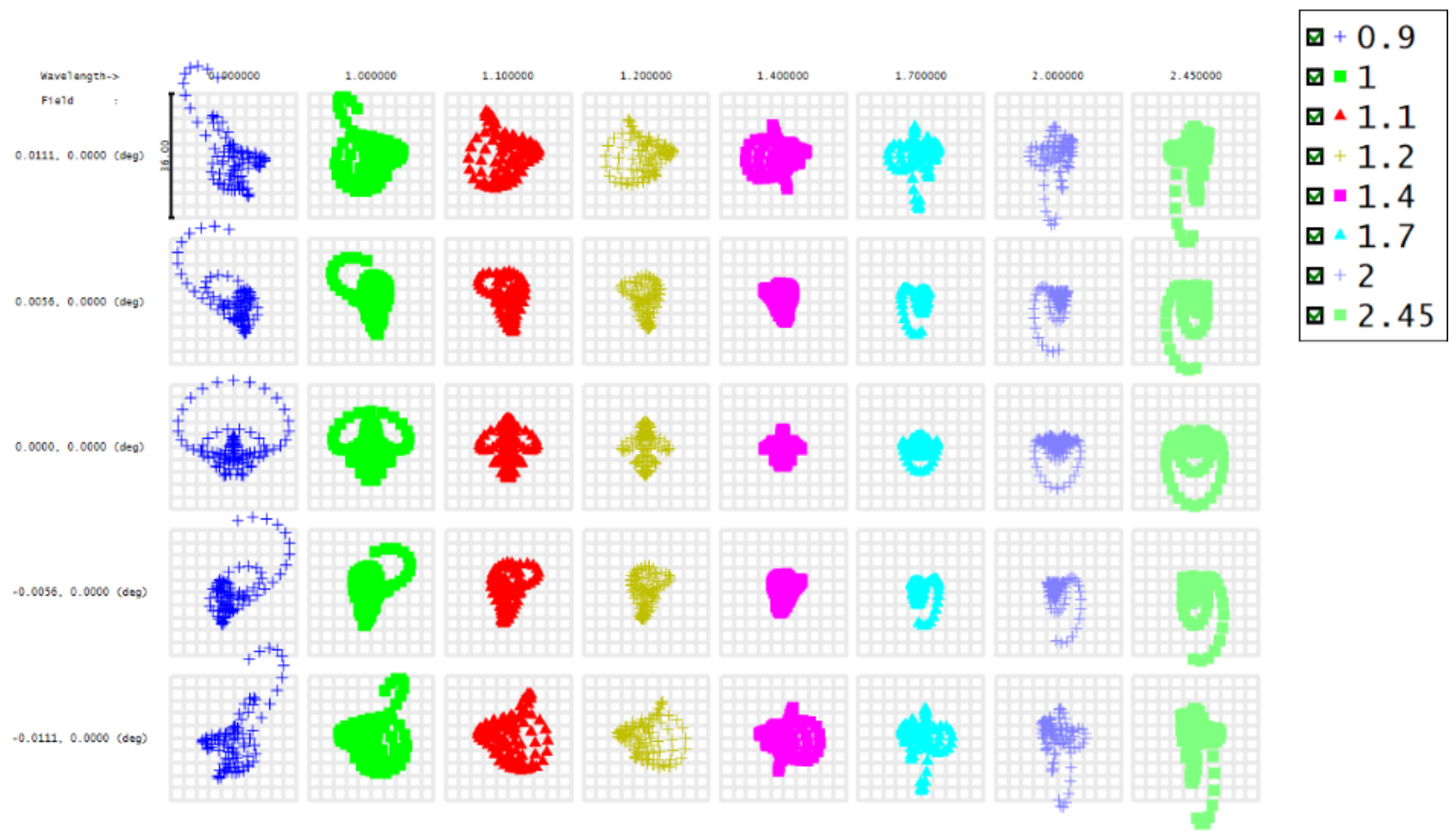

Figure 2. Spot diagrams for 5 fields (vertical) along the slit with the field center in the middle row and 8 wavelengths (horizontal) with 0.9 microns at the left and 2.4 microns at the right. The edge fields are 40 " from field center and the intermediate fields are 20". The box size for each spot is 36 microns, about 2 pixels on the detector. 


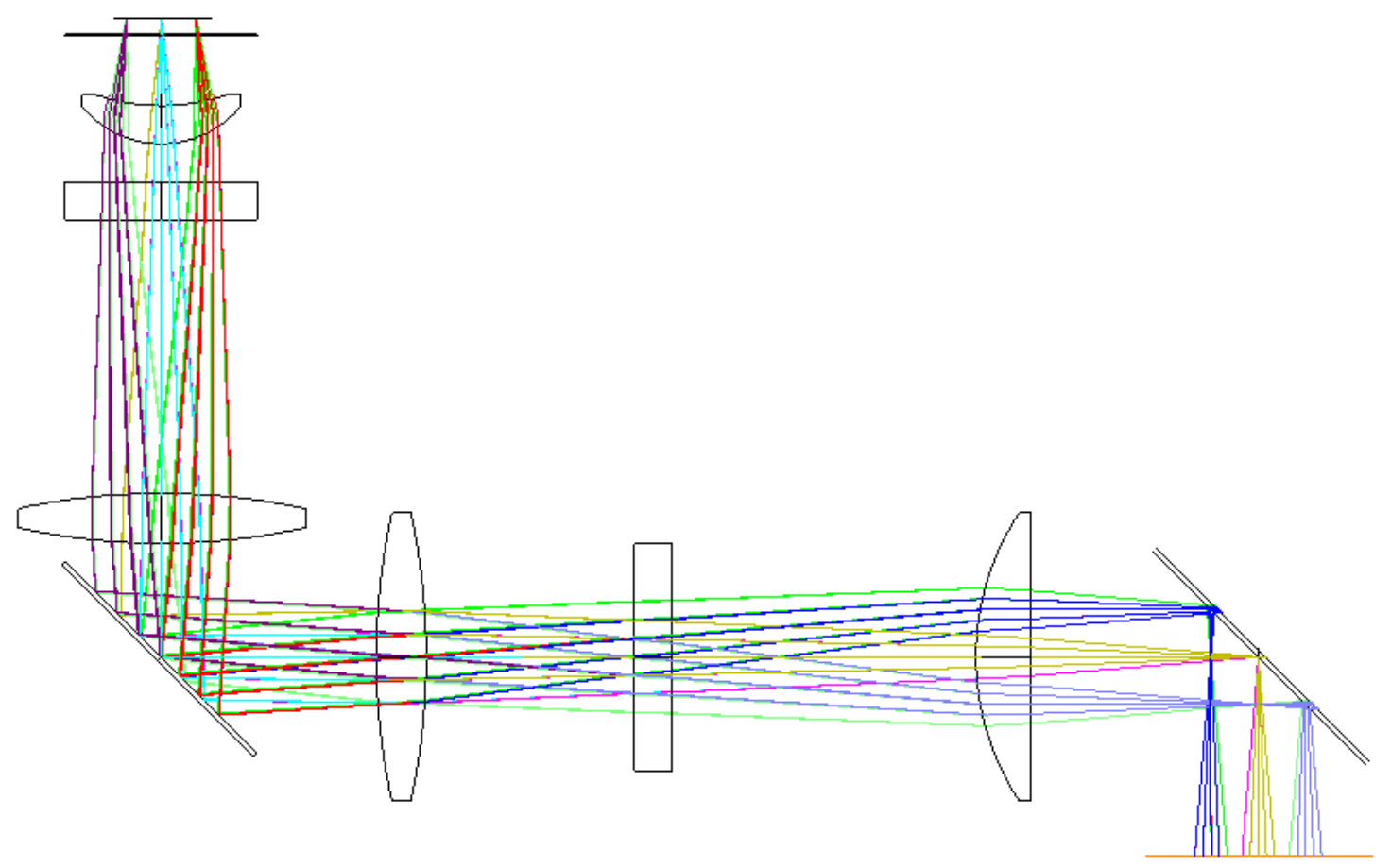

$100 \mathrm{~mm}$

Figure 3. Layout of the NIHTS slit-viewing optics. Light from the Offner relay and small fold mirror reflects from the slit jaws at lower right, proceeds through a field lens, dewar exit window, two $\mathrm{BaF}_{2}$ biconvex lenses separated by a fold mirror, a filter, the meniscus field flattener, and finally to the Xenics slit-viewing camera.
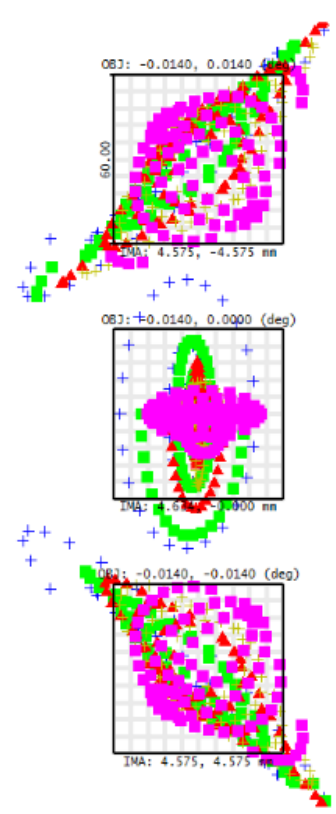
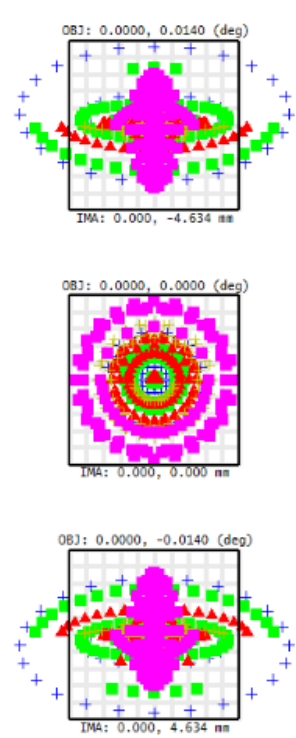

田 0.9

$\square=1$

$\square 1.1$

$\square+1.2$

$\square=1.35$

Figure 4. Spot diagrams for 9 fields centered on the field center. The edge fields are 50" off center and the corner fields are $50 "$ off center in both directions. Rays are colored by wavelength as shown in the legend. The box size is 60 microns or 2 pixels on the detector. 


\subsection{Dichroic fold mirror}

A dichroic beamsplitter has been designed, fabricated, coated, and installed in the deployable fold mirror assembly for NIHTS, enabling simultaneous NIR spectroscopy and optical imaging with LMI. Its design requirements included a 3 arcmin-square reflected field-of-view, a small wedge angle on the back surface to reduce tilted-plate astigmatism in the through-beam, very flat surfaces for a large width/thickness aspect ratio optic, and a unique coating that reflects at high efficiency from 0.9-2.5 microns while efficiently transmitting from 0.4-0.7 microns to cover the Sloan g' and r' bands. In addition, there are very tight space constraints for such a mounted and translatable optic inside the DCT instrument cube. The image quality requirement for the transmitted beam was approximately $1 / 2$ pixel (15 micron pixels) RMS spot sizes to 1 arcmin radius, with $90 \%$ encircled energy within 1 pixel. The modeled PSF ellipticity at median seeing $(0.84 \operatorname{arcsec})$ was $2.5 \%$.

The above requirements translated into an elliptical fused silica mirror $190 \mathrm{~mm} \times 134 \mathrm{~mm} \times 12.7 \mathrm{~mm}$ thick (longaxis/thickness aspect ratio of 15), with a $0.147+/-0.004^{\circ}(15 \mathrm{arcsec})$ wedge angle, and surface flatness $<1$ fringe. Three mirrors were fabricated of Dynasil by Zygo Corp., meeting the very precise wedge angle and flatness tolerances (the latter at $<0.5$ fringe).

Operating two instruments simultaneously on the same telescope creates a problem of simultaneously focusing both instruments. Focusing must accommodate the $4.3 \mathrm{~mm}$ focus shift introduced by the beamsplitter itself and also small filter-dependent LMI focus shifts. This is straightforward in the DCT environment. We focus LMI first by moving the DCT's secondary mirror, then focus NIHTS by moving its fold mirror in the DCT instrument cube. This has the effect of changing focus, but also moving where the NIHTS slit maps into the LMI field of view. Because LMI has such a wide field there is plenty of room to accommodate this.

\subsection{Coatings}

The AR coatings for the spectrograph and slit-viewing optics were multilayer coatings done by Infinite Optics. We had some adhesion problems with the $\mathrm{BaF}_{2}$ lenses in the slit-viewing optics, most likely due to the high thermal expansion coefficient of $\mathrm{BaF}_{2}$, but otherwise the coatings have performed admirably. The average 2-surface reflection loss of the $\mathrm{ZnS}$ prism over the NIHTS wavelength range is only $2.2 \%$. The worst-case loss is $5 \%$ at the short wavelength end of the range. For the fused silica windows and the fused silica slit-viewing lenses, the average 2-surface reflection loss is $2.6 \%$ and the worst-case loss is $4 \%$, again at short wavelengths. For the $\mathrm{BaF}_{2}$ lenses these values are $2.6 \%$ and $5 \%$, the worst case again being at short wavelengths.

Infinite also did our gold coatings. The coating included a 10 Angstrom layer of Inconel to improve the coating robustness, but the reflectivity is the same as a bare gold coating.

The dichroic fold mirror coatings were challenging as the optics reflect in the NIR while transmitting at optical wavelengths, opposite to most configurations. Multi-layer dielectric coatings were applied by Omega Corp. to the front surfaces, achieving 90.1\% average transmission (0.405-0.710 microns) and 96.8\% average reflectance (0.9-2.5 microns). A wide-band antireflection coating was applied to the back surface to minimize optical ghosting, having average reflectivities of $3.5 \%$ and $4.0 \%$ over these two bandpasses, respectively.

\section{MECHANICAL DESIGN}

Many features of the mechanical design have been described previously ${ }^{3}$ so we will focus the present discussion on updates to that paper and design elements not covered there. An important feature to reiterate is that NIHTS has (almost) no moving parts. The exception to this is a focus and tilt adjustment built into the detector mounting bracket. All other optical elements are precision machined and placed in their appointed locations so no other alignment adjustments are needed or possible.

The mounting structure for the Sunpower GT cryocoolers constrains the cooler body in all rotational degrees of freedom and in displacement in both directions perpendicular to the motion of the internal piston in the cooler. The only degree of freedom remaining is axial motion, to allow for possible uncompensated vibrational motion of the cooler body. All of our coolers use the passive tuned mass damper provided by Sunpower. This mounting structure was originally designed for LMI in 2012 and doesn't allow for the now-available cooling jacket for the main body of the cryocooler. The section of the mounting structure attached to the cooler connects to the section of the mount that is attached to the dewar wall via steel flexures and silicone dampers. A vacuum bellows connects the vacuum flange on the cooler to a mating flange 
on the dewar wall, and a flexible cold strap between the cooler's cold finger and the internal rigid cold straps provides compliance for the residual vibrational motion of the cooler. With this approach we have had no trouble with vibration conducted into the instrument on any of the instruments we currently operate, including NIHTS.

We were somewhat concerned about possible resonant coupling of the cryocooler to individual components on the optical bench. To check for this we measured the resonant frequencies of the various optical mounts, with optical elements in them, using a magnetic phonograph cartridge as a vibrational transducer. This simple approach was effective. The two lowest frequency components were the prism and return mirror in their mounts. The return mirror showed a relatively disorganized oscillation at $250-300 \mathrm{~Hz}$ that damped out in about 3 cycles. The prism was similar, oscillating at $\sim 400 \mathrm{~Hz}$ and damping out in $\sim 6$ cycles. We have seen no evidence of optics oscillation in the completed instrument.

The internal clearances between the floating shield and the optical bench, on one side, and the dewar wall, on the other side, were sufficiently small that we were concerned about possible thermal shorts. We added wires brought out through pins on a hermetic connector that were attached to the bench and shield so that we could verify the absence of a thermal short after assembly was complete but before beginning to cool the instrument.

Once all of the mechanical components were completed the interior surfaces and components of the instrument were painted with Aeroglaze Z306 and baked for 24 hours at $65 \mathrm{C}$ to harden the surface and drive off the volatile constituents of the paint. We found the online description of this process from the University of Virginia group ${ }^{5}$ to be very helpful. The external surface of the optical bench, its cover, the floating shield, and dewar interior wall were all left bare for low emissivity. Exterior surfaces of the instrument were black anodized.

The mounting interface between the instrument and the DCT instrument cube contains the slit-viewing camera and its reimaging optics. The mount is centered on the spectrograph entrance window while the slit-viewing optics are offset. The amount of space available wasn't quite enough to fit the Xenics camera in, so the length of the mounting interface was increased slightly. As a result NIHTS is not exactly at the zero spherical aberration optimal focus position of the DCT, and the clearance between the camera and the instrument cube is very small.

The NIHTS electronics are mechanically mounted in two locations. The detector controller and its power supply, and both cooler controllers and their power supplies, are mounted on a plate attached to the top of the cryostat, covered by a 0.050 " thick sheet metal shroud. A measure of the tightness of the fit of NIHTS on the DCT instrument cube is that NIHTS fits without the sheet metal shroud, but a few bolts holding the cube to the telescope needed their heads ground down slightly to allow NIHTS to fit with its electronics cover installed! The remaining electronics, including a Lakeshore 325 two-channel temperature controller, Lakeshore 218 8-channel temperature monitor, the vacuum gage controller, and various smaller items are contained in a separate package (the so-called Lakeshore stack) mounted to the telescope independently and cabled to the instrument.

NIHTS is installed or removed in three parts: the instrument itself $(140 \mathrm{lbs})$, the instrument-mounted electronics $(50 \mathrm{lbs})$, and the Lakeshore stack (60 lbs). Each of these parts can be handled by two people.

\section{CRYOGENICS}

\subsection{Cryocoolers}

NIHTS uses Sunpower Cryotel GT cryocoolers with liquid-cooled heat rejection surfaces like the other Lowell DCT instruments. Thermal modeling indicated that the NIHTS thermal load would be on the edge of a single cooler's capability $^{3}$, so our design allowed for either one or two coolers. The first cool-down used a single cooler but its ability to cool the detector sufficiently was marginal so we elected to use two coolers. Cooler 1 primarily cools the detector and getter but also provides some cooling to the optical bench. Cooler 2 cools the bench only. There is very little crosstalk between the coolers and this arrangement has worked well.

\subsection{Cold straps}

The cold strapping for cooler 1 is complicated. It is routed from the cooler's cold finger through a flexible cold strap, a heavy solid cold strap, then a feed-through to the inside of the optical bench, then another solid cold strap to points near the detector and getter, and finally through separate braid cold straps to the detector and getter. 
The flexible cold strap between the vibrating cold finger of cooler 1 and the rigid internal cold strap was a challenge. Combining flexibility and high thermal conductivity while fitting into a very limited volume and putting no side load on the cooler's cold finger was the key problem. Figure 5 shows a view of the final cold strap. Each of the 12 flexible joints use 7 sheets of 0.002 " copper shim with 0.005 " indium sheet interleaved and clamped together. The indium sheet is only present under the clamps. The thin copper shim parts were made for us by United Western Enterprises by photoetching.

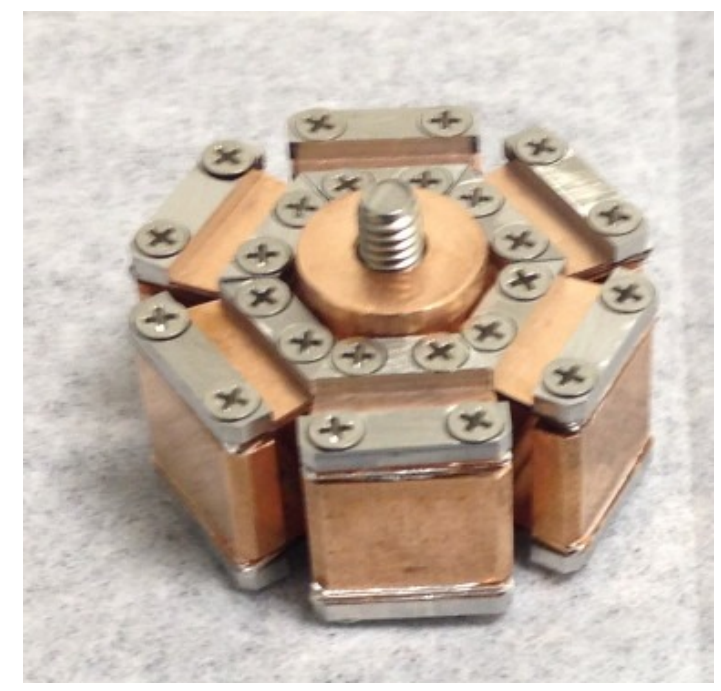

Figure 5. Flexible cold strap between cooler 1 and the rigid internal cold strap leading to the detector. The 8-32 screw in the center indicates the scale of the image.

The feed-through from the outside to inside of the optical bench had to be light tight but thermally insulated. This was accomplished by supporting a copper rod by G10 disks together with a labyrinth seal consisting of two aluminum disks (supported externally at the ends of the feed-through) and a copper disk soldered to the middle of the central rod.

Tuning of the conductivity of the cold straps and their connections took a few cool-downs with strategically located temperature sensors to allow the heat flows to be determined. Some joints needed indium sheet to ensure high conductivity while others intentionally left it out, or even used thin insulating G10 shims. The ability to set the temperature of the cold tip of the Sunpower coolers provides a very important degree of freedom. In normal operation cooler 1 is set to $55 \mathrm{~K}$, cooler 2 to $65 \mathrm{~K}$, and the detector operates stably at $75 \mathrm{~K}$ with the bench (unregulated) at $90-100 \mathrm{~K}$ depending on the season. Cooler 1 operates near its 70 watt minimum power level while cooler 2 operates near the middle of its operating power range.

\subsection{Getter and getter heater}

NIHTS includes a getter with an internal heater for vacuum baking the getter in situ. The getter is a sintered bronze muffler filled with charcoal that has been washed and baked, and includes a $25 \Omega$ heater. During baking the getter regulates at $333 \mathrm{~K}(60 \mathrm{C})$ and the detector temperature is monitored to ensure it doesn't exceed $323 \mathrm{~K}$ (50C). Most of the good from baking the getter happens in 12 hours, well before there is any risk of the detector being too warm. The ability to bake the getter in place has been very helpful.

\subsection{Temperature sensors and control loops}

NIHTS has two temperature control loops, one for the detector and one for the getter heater, each with a platinum RTD temperature sensor and heater resistor connected to the Lakeshore 325 controller. The Sunpower coolers have RTD sensors that are required for stabilizing the cold tip temperature, and these can be read from the cooler controllers. There are 8 additional sensors read by the Lakeshore 218 unit: 2 for the cooler heat rejection surfaces and one each for the dewar wall, floating shield, optical bench, prism, the detector mounting bracket, and the inner cold strap. The scripts described in Section 6.3 keep track of this data along with other relevant data sources. 


\subsection{Cool-down and warm-up behavior}

Cooling NIHTS takes about 3-4 days, limited by the rate at which cooler 2 can extract heat from the optical bench. Early in the cool-down we try to baby-sit the instrument to prevent the detector from cooling faster than $1 \mathrm{~K} /$ minute, but after the first 30 minutes or so this risk disappears and the instrument can be left unattended.

Controlled warm-up takes a similar length of time if the dewar is kept on a vacuum pump as it warms. Again we try to baby-sit during the first part of the warm-up to keep the detector from warming faster than $1 \mathrm{~K} /$ minute. Uncontrolled warm-ups are faster and cause more anxiety. The most severe instance of this was when cooler 1's controller failed and cooler 1 stopped operating. This occurred shortly after the daily plot was emailed out and was unrecognized for about 24 hours. The warm-up was essentially complete in 36 hours. No damage occurred and after pumping and cooling NIHTS was working normally again.

\section{ELECTRONICS}

\subsection{Detector controller}

NIHTS uses a detector controller from Astronomical Research Cameras, Inc. This controller uses Gen III timing and PCI boards, the ARC-32 clock driver board, and two ARC-42 2-channel video boards mounted in a 6-slot controller housing. There is no utility board because thermal control and monitoring is implemented independently as described in section 4.4. We use the HAWAII DSP code from ARC, modified to reflect our particular device and wiring approach.

Generally speaking, the controller has been trouble-free but we did run into a problem that also was also seen in the DeVeny spectrograph's controller. Both instruments have spectral calibration arc lamps that operate at high voltage by means of a transformer. When turning the lamp off the resulting surge on the power line often caused the controllers to be reset. This was due to inductive coupling to the reset and power supply over-temperature lines that are part of the DC cable running from the power supply to the controller. Since we never use the reset button in the power supply, and the DCT dome never gets very hot, we simply cut these lines and the problem was resolved.

\subsection{Cold pre-amp board}

The HAWAII-1 detector is mounted on a cold PC board with four small daughter boards each containing a pre-amplifier for one of the four outputs. The pre-amplifier design was suggested by Mark Farris (Teledyne) when we carried out our detector acceptance testing. It is a specific implementation of the general concept presented in Figure 2 of Finger, et al. ${ }^{6}$. Our preamp is based on the TL2272AID CMOS op-amp and is configured for a gain of 2. This op-amp has a specified minimum operating temperature of $-40 \mathrm{C}$ but functions at $\sim 80 \mathrm{~K}$. We initially operated it with $\pm 5 \mathrm{~V}$ rails but found that the upper rail needed to be raised to $+6.6 \mathrm{~V}$ to avoid very slow step response that manifested as long horizontal trails following bright pixels.

\subsection{Cooler high-temperature cutoff board}

The Sunpower first and second generation cooler controllers do not include a protection circuit in case the coolant flow for the cooler's heat rejection surface is interrupted. This was a likely failure mode with the consequence that the cryocooler would overheat in a few minutes and be destroyed. We therefore developed a small PC board based on an LM339 op-amp that implemented a comparator with a large hysteresis loop. The comparator's input was a $100 \Omega$ platinum RTD bonded to the heat rejection surface of the cryocooler, and the feedback resistors were chosen to turn off the cooler at a temperature of $60 \mathrm{C}$ and turn it back on once the temperature dropped to $45 \mathrm{C}$. For the first generation coolers a solid-state relay was used to interrupt the AC input to the power supply that operates the cooler. The secondgeneration controller has a logic level input to turn off the cooler that was used instead. Our temperature limits are conservative but are safe and do the job. When the coolant is interrupted, which usually happens due to maintenance work, the heat rejection surface's temperature limit cycles with a period of a few minutes. The cooler is none the worse for wear but the item being cooled, the detector or optical bench in the case of NIHTS, slowly warms up.

\subsection{Other}

Both Sunpower cooler controllers, both Lakeshore units, and the vacuum gage controller have serial interfaces. To communicate with them we use a dedicated MOXA IA5450A-T 4-port networked serial device server for NIHTS and one channel of the same type of unit that is also used by LMI. This device has a wide operating temperature range (down to -40C), has been very reliable, and is sufficiently flexible to operate with a wide variety of serial devices. 
A UVP Pen-Ray xenon lamp has been installed in the bottom of the DCT instrument cube for NIHTS spectral calibration. To use it the instrument cover, which is white on the inside, must be closed and the NIHTS fold mirror must be extended. Light from the lamp scatters off the instrument cover whereupon it follows the normal optical path into the instrument. The light path is not the most efficient due to other constraints in the instrument cube so exposure times are longer than we would like, up to 120 seconds.

There are two USB peripherals in the NIHTS electronics: the Xenics slit-viewing camera and a USB-controlled PowerUSB power strip. These are connected to the controlling computer, a Mac Mini located in the computer room, by an Icron USB 2.0 Ranger 2224 fiber optic USB extender. The PowerUSB controls the Xenics camera, the ARC controller for the NIHTS detector, and the xenon lamp for wavelength calibration.

\section{SOFTWARE}

\subsection{LOIS/LOUI}

Generally speaking, the DCT instruments are operated by a stripped-down version of the LOIS control software, with its GUI components removed, and LOUI, a java GUI based on the Eclipse Rich Client Platform. These communicate through the Apache ActiveMQ message broker along with many other DCT software components ${ }^{7}$.

NIHTS provided some challenges to the CCD-centric LOIS/LOUI paradigm that were partially met by modification of LOUI to provide the capability of displaying difference images. Similarly, LOIS was modified to be able to read out either the raw reset and read images of a reset-read-integrate-read imaging sequence or the correlated double sample difference of the post-integration and pre-integration reads. This arrangement provided the necessary underpinnings for a higher-level control software entity to orchestrate the necessary telescope motions and image acquisition sequences to generate the ABBA dither sequences commonly used in infrared astronomy. LOUI included a simple command sequencing module, without branching or looping capability, that could accomplish this but it was decided that a better approach was to create a new software layer that would command LOIS through the message broker. LOUI was retained in modified form for its spectrum display and analysis capabilities as well as its facility summary display, but its image acquisition views were removed in order to avoid bookkeeping problems for the higher-level code that normally commands LOIS to take images.

\subsection{High-level control}

The two primary interfaces that observers use to control the NIHTS slit viewing camera and NIHTS spectral channel are an iPython control session in a terminal window and a Python GUI for slit viewing camera image display and analysis. The observer issues commands in the iPython session to control exposures on the slit viewing camera and the NIHTS spectral channel (e.g. defining the type of exposure, exposure time, setting the desired slit). The slit viewing camera is a USB device on the user interface machine, so these commands are local. The spectral channel is commanded by LOIS commands sent through the message broker. A camera control GUI is currently in the works to replace the iPython terminal window. Since this software remains under development it will be described in more detail in a future paper.

\subsection{Pipeline data reduction}

Mike Cushing is modifying the Spextool data reduction software package ${ }^{8}$ so that it can work with NIHTS data. Several challenges were encountered in developing NIHTS compatibility in Spextool. The stepped slit widths of NIHTS complicated this process because the slit width is determined by details of the telescope pointing rather than by a mechanism. The large (factor of $\sim 4 \mathrm{x}$ ) change in resolution per pixel across the wavelength range of the instrument made it difficult to determine a dispersion solution. Curvature along both the spectral and spatial direction further complicated assignment of a dispersion solution. Overall, these problems have largely been addressed. The modification of Spextool is almost complete and will be described in a subsequent publication.

\subsection{Cryo-vacuum monitoring and alarms}

We use a set of shell scripts controlled by the unix cron utility to query the cooler controllers and Lakeshore units once per minute and the vacuum gage every 5 minutes through the MOXA units to monitor the cryogenic health of the instrument. We have found that the MOXA units have trouble if two serial ports are queried at the same time so cron staggers the queries through each minute. These scripts create logs of the cryo-vacuum data and send email status updates and alerts. A separate Python script creates a plot spanning the two most recent days showing all of the relevant 
data on one graph. The legend on the plot also includes numerical values for the current conditions. This plot is continuously updated, is accessible using a web browser, and provides an instant overview of the instrument's status.

\section{PERFORMANCE}

Initial commissioning work began with the first installations of NIHTS on the DCT in late 2015 and early 2016. Work was interrupted by a fold mirror collision in the DCT instrument cube after the scheduled downtime during the summer monsoons in 2016. Final commissioning observations, including simultaneous LMI operation, began after installation of the dichroic fold mirror in December 2017.

\subsection{Imaging and spectral performance}

The image scales of the spectrograph and slit-viewing camera were found to be 0.130 and 0.326 "/pixel respectively, in excellent agreement with their design values. The 8 spectrograph slit segments were 12" long for a total slit length of 96". The slit-viewing camera orientation maps the slit along the long dimension of the detector (320 pixels) so the slit image runs from side to side with the top of the slit being on the left side of the slit-viewing camera's image.

Focus runs on the telescope optimizing the image size as seen in the slit-viewing camera and the spectrum width seen in the spectrograph show a focus differential of approximately 70 microns of DCT secondary mirror position. This is a relatively small amount, and focusing at the midpoint of these positions reduces the defocus in both focal planes to a negligible value. The image size in both the spectrograph and slit-viewing camera are seeing limited except toward the corners of the slit-viewing camera's field, as expected.

Xenon and planetary nebula line widths observed on the telescope show FWHM values of 3.4 pixels for the 2 pixel slit, 4.9 pixels for the 4 pixel slit, and 6.7 pixels for the 6 pixel slit. Moving the detector $1 / 3 \mathrm{~mm}$ farther from the prism would improve the spectrograph's focus, but we have held off on this until a more compelling reason arises to open the instrument. The narrowest slit would benefit most but it is rarely used, being only 0.26 " wide on the sky, and it suffers most from non-uniformity in the slit edges. Detector tilt, determined by the width of xenon lines across the spectrum, was ultimately reduced to $0.07^{\circ}$, a value below our adjustment threshold.

Dispersion modeling based on xenon lamp spectra and planetary nebula spectra agree with the Zemax model to an rms accuracy of 23 microns (1.24 pixels), and the NIHTS dispersion model is now incorporated in the Spextool pipeline. The spectrum is offset by about 50 pixels $(\sim 0.9 \mathrm{~mm})$ towards the blue and 32 pixels $(\sim 0.6 \mathrm{~mm})$ above its modeled position on the detector.

\subsection{Stray light}

We found two stray light problems, one in the spectrograph and one related to the slit-viewing optics. The stray light in the spectrograph was due to thermal emission from the slit-viewing optics entering the slit perpendicular to the spectrograph's optical axis and reflecting off the internal surfaces of the slit structure. This produced a severe ghost reflection of the thermal background appearing at shorter wavelengths. We reduced the amplitude of this reflection by a factor of 30 by painting the interior surfaces of the slit assembly with Aeroglaze Z306. Its amplitude after painting was $1 \%$ of the thermal background peak.

The slit-viewing stray light problem was caused by a grazing reflection of off-axis stars off a flat surface of the slitviewing optics that entered the spectrograph's entrance window. This was easily remedied with some flocking paper on the offending surface.

We tested the efficacy of the Offner re-imager's cold stop by placing a soldering iron at several radial positions near the edge of the DCT primary mirror in four azimuthal locations around the mirror. The mirror has a black baffle to define its optical edge that we used as the fiducial mark for this test. The cold stop appeared to be well centered and had the effect of reducing the signal from the soldering iron by about half at the edge of the DCT primary baffle and to zero by $100 \mathrm{~mm}$ outside the baffle's edge.

\subsection{Noise, gain, linearity}

We measured the gain and noise of the detector system using the usual photon transfer approach. Best results were obtained by subtracting pairs of correlated double sample (CDS) read-reset images at varying signal levels, obtained by varying exposure time with a fixed illumination level. The analysis was done on two 60x60 pixel subframes in order to limit the effect of spatial variation in the illumination. The analysis accounts for the fact that the variance of the 
difference of the two CDS images is twice the variance of a single CDS image. The biggest uncertainties in this exercise were the known short-exposure nonlinearity seen in HAWAII-1 detectors ${ }^{6}$ and our inability to obtain truly dark conditions for dark frames. The latter problem caused our read noise values to be spuriously high. Our best estimate of the gain is 24 electrons/DN with $\sim 110$ electrons read noise in a CDS image. The noise is substantially higher than expected. Part of the problem may be additional shot noise from background light in our "dark" images and part may be real. Increasing the gain of the pre-amp might help if reducing the noise becomes important. In most cases shot noise on the sky background exceeds the read noise so we have not pursued a noise reduction project to date.

We used the same dataset to estimate the linearity performance of the detector. It is linear to a few percent up to a signal level of $\sim 6000 \mathrm{DN}$ or $\sim 140,000$ electrons.

\subsection{Sensitivity}

Observations of spectrophotometric standard star Feige 110 were obtained through the $4 "$ slit to determine the overall instrument throughput and sensitivity. This star was selected from the STScI CALSPEC catalog ${ }^{9}$. The wavelengthaveraged throughput of the telescope, dichroic, and instrument was determined by comparing the catalog flux of this star to the measured $\mathrm{DN} / \mathrm{s}$ by the instrument. The net throughput of the system was measured to be approximately $40 \%$, ranging from $45 \%$ at 1 micron and decreasing to $30 \%$ in K-band at 2.2 microns. Scaling the measured flux of Feige 110 suggests that the instrument would achieve $1 \mathrm{DN} / \mathrm{s}$ in $\mathrm{H}-$ band for objects as faint as $\mathrm{H}=23$.

Sky brightness at near-IR wavelengths fluctuates significantly at the DCT's Happy Jack site. Representative values measured during the observations of Feige 110 indicate sky background levels of $\mathrm{J} \sim 12, \mathrm{H} \sim 11$, and $\mathrm{K} \sim 8$., integrated across their respective bandpasses and a $\sim 1.5$ " seeing disk.

\subsection{LMI operation with NIHTS}

On-sky, the deployed dichroic fold mirror transmits an optical beam to LMI with an oval-shaped FOV of about $6.5 \times 4$ arcmin. This provides the capability to obtain simultaneous visible wavelength photometry while collecting NIHTS spectra. Over this field the flat field illumination is uniform to better than $5 \%$ and the throughput loss is $\sim 10 \%$ in the SDSS g' and r' bands, consistent with the dichroic coating transmission. Image quality of both the reflected and transmitted beams is good. Optical ghosting from a single internal reflection, offset by 120 arcsec from the stellar image, has been observed as predicted by Zemax, but so far only for stars whose signal fully saturates the LMI CCD. The area of this ghost is approximately $125 \operatorname{arcsec} \wedge 2$, and given the optical reflectivity of the two dichroic surfaces, should be brighter than the background of 50\% lunar phase sky only when $\mathrm{Mv}<\sim 9.5$.

NIHTS currently offers two primary modes of target acquisition: use of the Xenics slit-viewing camera and use of LMI through the dichroic. (A third approach, using a guide probe, hasn't been tried yet.) The slit-viewing camera can reasonably detect objects as faint as $\mathrm{H} \sim 17$ within several minutes through a combination of co-added exposures and capturing a sky background image for image differencing. Fainter objects require longer to detect with the slit-viewing camera and thus would impart a significant overhead on the observer. In these cases LMI can be used to detect and align the target onto the NIHTS slit. A pixel mapping from the LMI focal plane to the NIHTS slit mask can enable acquisition of targets (provided sufficient flux at visible wavelengths) down to the detection threshold of the NIHTS spectral channel.

To date operation of NIHTS with LMI has been done by operating the two instruments independently. The DCT software infrastructure is such that coordinated operation by a single high-level software entity is a possible avenue to pursue if the effort is justified by expected observing use cases.

\subsection{Reliability}

NIHTS has been on the DCT continuously since February, 2016 and the dewar has not been opened since then. We have had only two significant problems, largely thanks to the excellent backup power system at the DCT that effectively shields NIHTS from AC line power failures. The symptom of the first problem was corrupted images from the detector that appeared a few days before the planned summer 2016 shut-down for aluminizing the DCT primary mirror. This problem didn't respond to power cycling. NIHTS was warmed up, left alone in the lab for about 6 weeks, pumped, and when cooled and operated again the problem was gone and has never reappeared. We still don't know what caused it. The second problem was the failure of the controller for cooler 1, as described in Section 4.5. The Sunpower coolers themselves have been very reliable but we have had one first-generation controller (on LMI) and one second-generation controller (on NIHTS) fail since we began operating these coolers in 2012. 


\section{CONCLUSION}

NIHTS has been on the DCT for about 2 years and final commissioning is nearing completion. It has already been used for science observations and is now generally available for science, including remote observations by experienced users.

\section{ACKNOWLEDGEMENTS}

This work was supported by NASA Grant NNX09AB54G. The Mount Cuba foundation supported the dichroic beamsplitter coatings. We would like to thank James French, Charlie Burge, Liam Andrus, Steve Lauman, Jeff Gehring, and Glenn Hill for their contributions in the mechanical area, Saeid Zoonemat Kermani, Dyer Lytle, Len Bright, and the late Peter Collins for theirs in the software area, Rich Oliver for his help with the electronics, and Mike Cushing for his effort in adapting Spextool for NIHTS.

\section{REFERENCES}

[1] Levine, S.E., Bida, T.A., Chylek, T., Collins, P.L., DeGroff, W.T., Dunham, E.W., Lotz, P.J., Venetiou, A.J., and Zonnemat Kermani, S., "Status and performance of the Discovery Channel Telescope during commissioning", Proc. SPIE 8444, 844419 (2012).

[2] Bida, T. A., Dunham, E. W., Nye, R. A., Chylek, T., and Oliver. R. C., "Design, development, and testing of the DCT Cassegrain instrument support assembly", Proc. SPIE 8444, 844451 (2012).

[3] Bida, T. A., Dunham, E. W., Massey, P., and Roe, H. G., "First-generation instrumentation for the Discovery Channel Telescope", Proc. SPIE 9147, 91472N (2014).

[4] Warren, D. W., Hackwell, J. A, and Gutierrez, D. J., "Compact prism spectrographs based on aplanatic principles",Opt. Eng. 36, 1174-1182 (1997).

[5] http://faculty.virginia.edu/skrutskie/tspec/docs/tspec black_paint.pdf

[6] Finger, G., Mehrgan, H., Meyer, M., Moorwood, A.F.M., Nicolini, G., and Stegmeier, J., "Performance of large format $\mathrm{HgCdTe}$ and InSb arrays for low background applications", Proc. SPIE 4008, 1280-1297 (2000).

[7] Dunham, E.W., J.L. Elliot, T.A. Bida, P.L. Collins, B.W. Taylor, and S. Zoonematkermani, "HIPO Data Products", Proc. SPIE 7014, $70144 Z$ (2008).

[8] Cushing, M. C., Vacca, W. D., and Rayner, J. T., "Spextool: A Spectral Extraction Package for SpeX, a 0.8-5.5 Micron Cross-Dispersed Spectrograph", Pub. A.S.P., 116, 362-376 (2004).

[9] http://www.stsci.edu/hst/observatory/crds/calspec.html 